Supporting information for:

\title{
Probing the Cross- $\beta$ Core Structure of Amyloid Fibrils by Hydrogen-Deuterium Exchange Deep UV Resonance Raman Spectroscopy
}

\author{
Ming Xu, Victor Shashilov and Igor K. Lednev* \\ Department of Chemistry, University at Albany, SUNY, 1400 Washington Ave., Albany, NY, USA
} 12222

\section{Experimental Details}

Hen egg white lysozyme, $\mathrm{D}_{2} \mathrm{O}(99.9 \%$ atom D) were from Sigma-Aldrich (St. Louis, $\mathrm{MO}$ ). H-Gly-Gly-Gly-OH, H-Gly-Tyr-Gly-OH, H-Gly-Phe-Gly-OH were from Bachem Bioscience Inc. (King of Prussia, PA).

The lysozyme fibrils were prepared as previously reported. ${ }^{1,2}$ Briefly, $\sim 14 \mathrm{mg} / \mathrm{ml}$ of lysozyme solution ( $\mathrm{pH} 2.0$ ) was incubated at $65^{\circ} \mathrm{C}$ for 6 days. The fibrils (gelatinous phase) was collected by centrifugation at $16,100 \mathrm{~g}$ for $30 \mathrm{~min}$. The fibrils were washed with de-ionized $\mathrm{H}_{2} \mathrm{O}$ or $\mathrm{D}_{2} \mathrm{O}$ two times and collected by centrifugation at $16,100 \mathrm{~g}$ for 45 min. Fibrils were then suspended in either $\mathrm{H}_{2} \mathrm{O}$ or $\mathrm{D}_{2} \mathrm{O}$ and allowed to stand at room temperature for $>4$ hours before DUVRR measurements. Fibrils were suspended in $\mathrm{D}_{2} \mathrm{O} / \mathrm{H}_{2} \mathrm{O}$ mixture with $\mathrm{D}_{2} \mathrm{O}$ concentrations vary from $0 \%$ to $100 \%$ at $2 \%$ interval.

The supernatant, which consists of no detectable fibrils, ${ }^{1,2}$ was lyophilized. The lyophilized partially unfolded lysozyme intermediate was dissolved in either $\mathrm{H}_{2} \mathrm{O}$ or $\mathrm{D}_{2} \mathrm{O}$ in a concentration of $2 \mathrm{mg} / \mathrm{mL}$ for DUVRR measurement.

H-Gly-Gly-Gly-OH, H-Gly-Tyr-Gly-OH, H-Gly-Phe-Gly-OH were dissolved in either $\mathrm{H}_{2} \mathrm{O}$ or $\mathrm{D}_{2} \mathrm{O}$ for DUVRR measurement.

\section{Bayesian Data Analysis}

\section{Bayesian Approach}

The source separation problem is as follows

$$
\text { Data }=C \cdot S+E
$$

where $C$ is the concentration matrix, $S$ is the matrix of pure component spectra and $E$ is error where random or systematic. The matrix Data is known while the matrices $C$ and $S$ are to be estimated. The Bayes theorem for problem (1) is written as

$$
P(C, S \mid \text { Data, } I) \sim P(\text { Data } \mid C, S, I) \cdot P(C \mid I) \cdot P(S \mid I)
$$

where $P($ Data $\mid C, S, I)$ is the likelihood controlling the quality of fitting and $P(S \mid I)$ and $P(C \mid I)$ are prior probabilities for spectra and concentrations. Because finding either matrix $\mathrm{C}$ or $\mathrm{S}$ alone is enough for solving problem (1) the concentration matrix $\mathrm{C}$ is normally sought since it contains by far fewer elements. It was shown ${ }^{3}$ that in the case of uniform prior for concentration matrix and independent sources the probability of the concentration matrix is given by

$$
P(C \mid \text { Data }, I) \sim \int d s \cdot \prod_{i} \delta\left(\text { Data }_{i}-C_{i k} \cdot S_{k}\right) \cdot \prod_{l} p_{l}\left(s_{l}\right)
$$


which in the case of noise-free data reduces to the logarithmic probability

$$
P(C \mid \text { Data }, I)=\log (\operatorname{det}(W))+\sum_{l} \log \left(p_{l}\left(s_{l}\right)\right)
$$

where $W$ is the separation matrix such that $S=W \cdot$ Data.

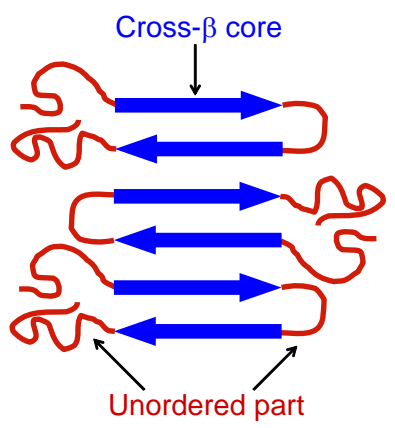

Figure S1. Schematic diagram of the structure of amyloid fibrils. Cross- $\beta$ core is shown in blue arrows; the unordered part is shown in red lines.

Fifty one Raman spectra of fibrils suspended in various concentration of $\mathrm{D}_{2} \mathrm{O}$ were used for the Bayesian data analysis. We deem the fibrils as the combination of the cross- $\beta$ core with the unordered part (Figure S1). The amide protons in the cross- $\beta$ core are not exchangeable because of extensive hydrogen-bonding interaction and the exclusion of water molecules (see main text). The amide protons in the unordered part are readily exchangeable. Therefore, there are three major species contributing to the spectrum of fibrils in the hydrogen-deuterium exchange (HDX) deep UV resonance Raman (DUVRR) experiment: the protonated cross- $\beta$ core, the protonated unordered part and the deuterated unordered part (Figure S2).

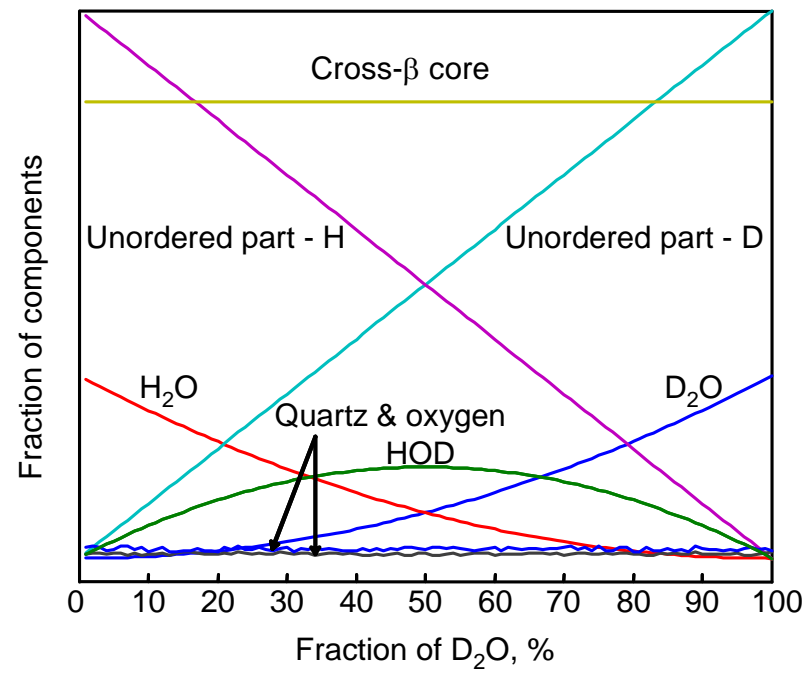

Figure S2. Anticipated concentration profile of pure components involved in the HDX DUVRR experiment.

\section{Incorporating prior information about the $C$ matrix}

The actual concentration matrix should have columns proportional to the columns of the matrix sketched in Figure S2. Eight hyper-parameters $\alpha_{i}$ then need to be estimated so that $C j=\alpha_{i} \cdot T_{i j}$ with $j=1: 8$ and $T$ is the template matrix shown in Figure S2. The parameters $\alpha_{i}$ account for the spectral fraction of each component in the mixtures (they are proportional but not equal to the physical concentrations and therefore must be found). For example, e.g. $\alpha_{1} / \alpha_{6}$ gives the spectral fraction of fibril core with respect to that of water and is proportional to the concentration of protein in samples. The concentration of protein is 
assumed to be equal in all samples since they were prepared based on the same stock solution.

As seen from Figure S2, contributions of quartz and oxygen are random and to be rigorous, 50 additional parameters need to be assigned for fraction of quartz in each sample and another 50 for oxygen. These are the nuisance parameters in the model. At this stage, however, we assumed constant but unknown fractions of quartz and oxygen in each spectrum. After all relevant parameters are found matrix least-squares will refine those small quartz and oxygen contribution in each sample. The posterior for the concentration matrix then takes the form

$P(C \mid$ Data,$I)=\log (\operatorname{det}(W))+\sum_{l} \log \left(p_{l}\left(s_{l}\right)\right)-\frac{m \cdot n}{2} \cdot \log \{\|(C-T \cdot \alpha)\|\}$

where $\alpha$ is diagonal matrix with parameters $\alpha_{i}$ on its diagonal and \|\| stands for Frobenius norm, $m$ is a number of experimental $(\mathrm{m}=50)$ spectra and $n$ is a number of pure components $(\mathrm{n}=8)$.

(a) Anticipating $\mathrm{H} 2 \mathrm{O}, \mathrm{D} 2 \mathrm{O}$, HOD contributions in each sample

$\mathrm{H}$-s and D-s from $\mathrm{H}_{2} \mathrm{O}$ and $\mathrm{D}_{2} \mathrm{O}$ molecules readily interchange to form mixed HOD molecules. If the total fraction of protons in the $\mathrm{H}_{2} \mathrm{O}-\mathrm{D}_{2} \mathrm{O}$ is $\mathrm{q}$ then the probabilities of forming $\mathrm{H}_{2} \mathrm{O}, \mathrm{D}_{2} \mathrm{O}$, HOD are as follows:

\begin{tabular}{llc}
\hline Probability & Possible Combinations & Statistical Weight \\
\hline $\mathrm{P}\left(\mathrm{H}_{2} \mathrm{O} \mid \mathrm{q}, \mathrm{I}\right) \sim \mathrm{q}^{2}$ & $\mathrm{H}-\mathrm{O}-\mathrm{H}$ & 1 \\
$\mathrm{P}\left(\mathrm{D}_{2} \mathrm{O} \mid \mathrm{q}, \mathrm{I}\right) \sim(1-\mathrm{q})^{2}$ & D-O-D & 1 \\
$\mathrm{P}(\mathrm{DOH} \mid \mathrm{q}, \mathrm{I}) \sim 2 *(1-\mathrm{q}) * \mathrm{q}$ & D-O-H, H-O-D & 2 \\
\hline
\end{tabular}

Simulated concentration profiles of $\mathrm{H}_{2} \mathrm{O}, \mathrm{D}_{2} \mathrm{O}, \mathrm{HOD}$ versus the concentration of $\mathrm{D}_{2} \mathrm{O}$ are shown in Figure S2.

(b) Anticipating unordered part $(H)$ and unordered part(D) contributions in each sample Fractions of N-H bonds and N-H bonds in the unordered part are proportion to the total concentration of H-s and D-s, respectively, i.e. they follow linearly the fraction of added $\mathrm{D}_{2} \mathrm{O}$ (Figure S2).

(c) Anticipating contribution of the other components

Each fibril sample was prepared from the same stock fibril material. This implies that the fraction of the $\beta$-sheet core with respect to the random coil part is constant across all samples, i.e.

$\operatorname{Frac}(\beta$-sheet $) /((\operatorname{Frac}(\operatorname{coil}(\mathrm{H}))+\operatorname{Frac}(\operatorname{coil}(\mathrm{D}))) \sim \mathrm{const}$

All spectra exhibit the admixture of quartz signal (spectra were recorded in a quartz tube) and atmospheric oxygen. Both fractions are random (Figure S2). Table S1 summarizes available prior information about all the components

Table S1. Prior information on the spectra and concentrations of components.

\begin{tabular}{|l|l|l|}
\hline Pure component & Spectrum & Concentration profile \\
\hline $\mathrm{H}_{2} \mathrm{O}$ & Known & Shape is known \\
\hline $\mathrm{HOD}$ & Known & Shape is known \\
\hline $\mathrm{D}_{2} \mathrm{O}$ & Known & Shape is known \\
\hline Unordered part, H substituted & Unknown & Shape is known \\
\hline Unordered part, D substituted & Unknown & Shape is known \\
\hline
\end{tabular}




\begin{tabular}{|l|l|l|}
\hline Fibril core & Unknown & Shape is known \\
\hline Quartz & Known & Small random contribution \\
\hline Oxygen (molecular) & Known & Small random contribution \\
\hline
\end{tabular}

\section{Incorporating prior information about the $S$ matrix}

In our model we have 5 pure spectra known from the experiment. It is straightforward to assign inner product of the known spectrum and the resolved spectrum for known components as their prior probabilities. Indeed, inner product equals 1 if spectra completely overlap and 0 if they have no overlapping regions. For the other three spectra $P\left(s_{i j}\right)$ was set proportional to the reciprocal of $s_{i j} . P\left(s_{i j}\right) \sim 1 / s_{i j}$. The total posterior probability then transforms into the following:

$$
\begin{aligned}
& P(C \mid \text { Data }, I)=P_{1}(C \mid \text { Data }, I)+P_{2}(C \mid \text { Data }, I) \\
& P_{1}(C \mid \text { Data }, I)=\log (\operatorname{det}(W))+\sum_{l} \log \left(p_{l}\left(s_{l}\right)\right)-\frac{m \cdot n}{2} \cdot \log \{\|(C-T \cdot \alpha)\|\} \\
& P_{2}(C \mid \text { Data }, I)=T \cdot\left(\sum _ { i = 1 } ^ { k 1 } \operatorname { l o g } \left(\text { inner } \left(s_{i},\right.\right.\right. \text { ref } \\
& \left.\left.-s_{i}\right)\right)-\sum_{k 1}^{m} \log \left(s_{i}\right)
\end{aligned}
$$

As seen from (6) assumption $P\left(s_{i j}\right) \sim 1 / s_{i j}$ for unknown spectra resulted in addition of

$-\sum \log \left(s_{i}\right) \quad$ known as a sparsity constraint. It controls the area of resolved spectra and eliminates extraneous bands appearing as admixtures from the other spectra.

It turned out in the course of optimization that resolved spectra had characteristic bands whose shapes were close to what we expected for these components. The space in between those bands contained admixtures from other components and /or noise. Alternatively, the characteristic bands in the sought spectra were obtained using pure variable approach ${ }^{4}$. The latter analyzes second derivative or fourth derivative spectra where even very overlapping bands are seen as distinct sharp peaks. The purest variable is such a wavenumber at which the contribution of an individual component to the Raman intensity is maximal while the contributions from the other components are minimal. For a Raman spectrum of each sample, the intensity at a particular purest variable is approximated to be proportional to the concentration of a corresponding individual component in the sample. Consequently, the matrix of the Raman intensities at all purest variables $C_{\text {int }}$ can be used as a concentration matrix $C$ of the components. The shapes of spectral band in normal (not second derivative) space are then found as

$$
S=\operatorname{Data}^{T} C_{\text {int }}\left(C_{\text {int }}{ }^{T} C_{\text {int }}\right)^{-1}
$$

Knowledge of characteristic bands allows modeling the unknown spectrum as a linear combination of the non-overlapping bands which are referred to as a dictionary bands ${ }^{5}$. A model dictionary spectrum is used in a similar fashion as known experimental spectra. The spectrum resolved at each iteration of the algorithm is compared to the dictionary spectrum by means of inner product. The space in between known bands in a dictionary spectrum is set equal to 0 . This means that any band of the resolved spectrum in the region one is not sure about will give zero contribution to the probability for the spectrum (they multiply by zeros in a dictionary spectrum) thus assigning equal probabilities to any 
spectral features in doubtful regions. Coefficients for contributions of various dictionary bands to model spectra were sought as additional parameters over the course of optimization.

\section{Sampling method}

The floating point genetic algorithm (GA) ${ }^{6}$ was used as a sampling methods. The GA thoroughly explores the parameter space thus getting around the local maxima and allows strict setting the prior range for optimized parameters

Accelerating the convergence

Augmented Data matrix and $C$ matrix were constructed at the initial stage of fitting. Namely, five known pure component spectra were put on the top of the Data matrix and five rows were added to the $C$ matrix with units on the diagonal and zeros otherwise. Thus the algorithm was forced to fit the augmented matrix using five augmenting spectra as five of eight components.

\section{Algorithm outline}

(1) Parameters $\alpha_{\mathrm{i}}$ are initialized, ranges of the parameters are constrained to the expected range.

(2) Reduce dimension of Data from $m=50$ to $n=8$ by SVD to produce matrix $D_{\text {trunc. }}$.

(3) For $i=1$ : itmax do

I. Sample parameters $\alpha$

(a) Calculate trial $C$ matrix as $C_{i j}=T_{i j} * \operatorname{diag}\left(\alpha_{j}{ }^{c o n c}\right)$, i.e. by multiplying columns of $\mathrm{T}$ by respective parameters, $T$ is a template matrix.

(b) Calculate dictionary spectra as $S_{d i c}=\alpha_{j}{ }^{\text {dict }}$. Band

(c) Add dictionary spectra multiplied by the small parameter $\lambda$ on the top of the augmented matrix (to speed up convergence), add rows with units on the diagonal and zeros otherwise to the augmented concentration matrix.

(d) Calculate resolved spectra by matrix least-squares using ' $\backslash$ ' Matlab operator

$$
S=\text { Data }_{\text {aug }}{ }^{T} C\left(C_{\text {aug }}{ }^{T} C_{\text {aug }}\right)^{-1} \quad \text { set } S(S<0)=\text { tol, tol }>0 \& \text { tol }<<1
$$

(e) Given spectra $S$ calculate the refined concentration matrix Conc

$$
\text { Conc }=\left(S^{T} \cdot S\right)^{-1} \cdot S^{T} \cdot \text { Data }^{T}
$$

using ' $\backslash$ ' Matlab operator for matrix pseudo-inverse

(e) Calculate posterior as $\mathrm{P}(\mathrm{C}, \mathrm{S} \mid$ Data, $\mathrm{I})=\mathrm{P}\left(\mathrm{C} \mid\right.$ Data, I) (eq. 6) $\frac{(m \cdot T)}{2} \cdot \log \|$ Data - Conc $\cdot S \|$

$$
\text { Separation matrix } W \text { is calculated as } \quad W=\left(\text { Data }_{\text {trunc }}{ }^{T} \cdot \text { Data }_{\text {trunc }}\right)^{-1} \cdot \text { Data }_{\text {trunc }}{ }^{T} \cdot S
$$

II. Sample a new set of parameters $\alpha$ and go through (a)->(e)

(4) End of the algorithm. 


\section{D-DUVRR analysis}

Two-dimensional correlation spectroscopy was utilized to reveal sequential order of change of deuterated and protonated parts of unordered fibrillar structures. The asynchronous 2D map showed that rise of intensities of deuterated amide bands was synchronous with intensity decrease in protonated bands. The latter proves the validity of our assumption about the shape of concentration profiles (Figure S2).

\section{Abstract factor analysis}

The abstract factor analysis of the HDX DUVRR data showed the presence of two significant components despite the presence of three spectroscopically distinguishable species. This finding implies that the actual concentrations of the fibrillar $\beta$-sheet core, and protonated and deuterated unordered parts followed the anticipated scheme (Figure S2), which has only two significant components because of the linear correlation between the concentration profiles.

\section{References}

1. Xu, M.; Ermolenkov, V. V.; He, W.; Uversky, V. N.; Fredriksen, L.; Lednev, I. K., Lysozyme fibrillation: Deep UV Raman spectroscopic characterization of protein structural transformation. Biopolymers 2005, 79, (1), 58-61.

2. $\quad \mathrm{Xu}, \mathrm{M}$; Shashilov, V. A.; Ermolenkov, V. V.; Fredriksen, L.; Zagorevski, D.; Lednev, I. K., The first step of hen egg white lysozyme fibrillation, irreversible partial unfolding, is a two-state transition. Protein Science 2007, 16, (5), 815-832.

3. Knuth, K. In Bayesian Source Separation and Localization, SPIE'98 Proceedings: Bayesian Inference for Inverse Problems, SPIE San Diego, July, 1998; San Diego, July, 1998; pp 147-158.

4. Windig, W.; Gallagher, N. B.; Shaver, J. M.; Wise, B. M., A new approach for interactive self-modeling mixture analysis. Chemometrics and Intelligent Laboratory Systems 2005, 77, 85-96.

5. Zibulevsky, M.; Pearlmutter, B. A., Blind Source Separation by Sparse Decomposition in a Signal Dictionary Neural Computation 2001, 13, 863-882.

6. Elliott, L.; Ingham, D. B.; Kyneb, A. G.; Mera, N. S.; Pourkashanian, M.; Wilson, C. W., Genetic algorithms for optimisation of chemical kinetics reaction mechanisms. Progress in Energy and Combustion Science 2004, 30, 297-328. 